\title{
El rol mediador de la implicación escolar sobre el apoyo del profesorado y la satisfacción con la vida
}

\author{
Estibaliz Ramos-Díaz, Arantzazu Rodríguez-Fernández, Lorena \\ Revuelta e Inge Axpe \\ Universidad del País Vasco/Euskal Herriko Unibertsitatea (España)
}

\begin{abstract}
La visión de la escuela como un entorno promotor del bienestar personal ha generado un gran interés en el ámbito de la investigación psicoeducativa por el estudio de variables relacionadas con el desarrollo positivo del alumnado. Este trabajo analiza mediante un modelo estructural tanto la influencia directa como indirecta del apoyo del profesorado a través de la implicación escolar sobre la satisfacción con la vida. Participaron en el estudio 1250 estudiantes de Educación Secundaria Obligatoria, de entre 12 y 15 años $\left(M_{\text {edad }}=13.72\right.$, $D T=1.09$ ), seleccionados aleatoriamente en la Comunidad del País Vasco, de los cuales el $49 \%$ eran chicos mientras que el $51 \%$ eran chicas. Los resultados confirman la influencia que el apoyo del profesorado ejerce sobre la satisfacción con la vida a través de la implicación escolar $\left(\chi^{2}(51)=219.743, p<.001 ; \mathrm{GFI}=.972 ; \mathrm{CFI}=.965 ; \mathrm{TLI}=.954 ; \mathrm{SRMR}=.031\right.$; RMSEA=.051). Para finalizar, se discuten los resultados y sus implicaciones, así como las perspectivas futuras de investigación.
\end{abstract}

Palabras clave: Apoyo percibido del profesorado, implicación escolar, satisfacción con la vida, secundaria, adolescentes.

The mediating role of school engagement on perceived teacher support and satisfaction with life. The view of the school as a enviromental promoter of personal well-being has raised great interest in the study of variables related to positive development of students in the field of psychoeducational research. This study aims to test a structural model which posits both direct and indirect influence of perceived teacher support through school engagement on satisfaction with life. The study comprised 1250 students of compulsory secondary education aged between 12 and 15 years $\left(M_{a g e}=13.72 ; S D=1.09\right)$ randomly selected in the Basque Country, of whom 612 (49\%) were male and $638(50 \%)$ female. The results confirm the influence exerted by perceived teacher support on satisfaction with life through school engagement $\left(\chi^{2}(51)=219.743, p<.001 ; \mathrm{GFI}=.972 ; \mathrm{CFI}=.965\right.$; TLI=.954; $\mathrm{SRMR}=.031$; RMSEA=.051). Finally, the results and their implications, as well as the future research perspectives are discussed.

Keywords: Perceived teacher support, school engagement, satisfaction with life, secondary, adolescents.

Correspondencia: Estibaliz Ramos-Díaz. Facultad de Educación y Deporte. Departamento de Psicología Evolutiva y de la Educación. Universidad del País Vasco. C/ Juan Ibáñez de Sto. Domingo, 1. C.P.: 01006. Vitoria-Gasteiz (España). E-mail: estibaliz.ramos@ehu.eus 
Una de las finalidades principales del sistema educativo, más allá de la transmisión de conocimientos académicos, es el desarrollo de competencias emocionales y sociales en el alumnado que contribuyan a un mayor bienestar personal, o lo que es lo mismo, a un desarrollo integral del alumnado (Pertegal, Oliva, y Hernando, 2010). Esta consideración de la escuela como un entorno promotor del desarrollo saludable ha generado un renovado interés por la psicología positiva y su aplicación al ámbito educativo (Froh, Huebner, Youssef, y Conte, 2011; Kristjánsson, 2012), dejando atrás la investigación psicoeducativa tradicional centrada en las limitaciones y conductas problema de los escolares adolescentes. En este nuevo contexto, el enfoque de desarrollo positivo en el ámbito escolar (educación positiva) ha sido definida como la integración de los principios básicos de la psicología positiva relacionados con la promoción de los activos escolares o fortalezas del alumnado a través de prácticas e intervenciones educativas que mejoran el ajuste psicológico de los estudiantes (Noble y McGrath, 2015).

Uno de los indicadores de bienestar subjetivo o ajuste psicológico más importantes es la satisfacción con la vida (Diener, 2009), entendida como la valoración positiva de la propia vida considerada en su conjunto (Rodríguez y Goñi, 2011). De manera similar a los estudios en población adulta, se ha demostrado que la satisfacción con la vida es un indicador de adaptación general en múltiples dominios de la vida también en la adolescencia (Lewis, Huebner, Malone, y Valois, 2011). Esta variable se expresa en forma de juicio global que la persona realiza sobre su trayectoria vital (Diener, Oishi, y Lucas 1994; Campbell, Converse, y Rogers, 1976). Aunque la investigación sobre la temática de la felicidad ha adquirido un escaso protagonismo en los estudios con adolescentes (Casas, 2011), el conocimiento de las consecuencias negativas que derivan de un desarrollo psicológico inadecuado ha generado un gran interés por el análisis de elementos relacionados con el ajuste psicológico óptimo del adolescente (Fuentes, García, Gracia, y Alarcón, 2014). Mientras que factores de carácter intraindividual explican un alto porcentaje de la varianza del bienestar subjetivo, se sabe asimismo que factores ambientales en términos de apoyo social contribuye a una percepción de mayor de satisfacción con la vida (Proctor, Linley, y Maltby, 2009).

El apoyo social percibido es una variable predictora del ajuste adolescente (Azpiazu, Esnaola, y Sarasa, 2015; Chu, Saucier, y Hafner, 2010; Fernández-Zabala, Goñi, Camino, y Zulaika, en prensa), y se entiende como la percepción individual de que la red social proporcionará un soporte adecuado en momentos de necesidad (Lakey y Scoboria, 2005). Se trata de un fenómeno complejo que comprende un conjunto de elementos en interacción y que evoluciona en gran medida a lo largo de la adolescencia (Cohen, 2004). Un amplio cuerpo de evidencia empírica confirma que la existencia de relaciones positivas entre profesorado y alumnado contribuyen a una amplia gama de resultados positivos relacionados con el bienestar del estudiante (Noble y McGrath, 2015). Concretamente, se ha demostrado que la percepción por parte de los estudiantes de que son apoyados por la 
figura docente correlaciona positivamente con la satisfacción con la vida e incluso contribuye significativamente a la predicción de este índice de desarrollo positivo adolescente (Guess y McCane-Bowling, 2016). Se sabe asimismo que el apoyo emocional del profesorado está positivamente relacionado con el ajuste personal de los estudiantes y negativamente relacionado con síntomas de desajuste psicológico tales como problemas escolares, problemas de internalización, falta de atención, hiperactividad y síntomas emocionales generales (Tennant et al., 2014). En definitiva, la conexión estrecha y positiva entre el estudiante adolescente y el profesor se encuentra consistentemente relacionada con el bienestar emocional, independientemente de aspectos como la edad o el rendimiento percibido en la escuela (García-Moya, Brooks, Morgan, y Moreno, 2014).

Por otro lado, existe evidencia del impacto directo de las relaciones alumnado-profesorado en el desarrollo positivo de los adolescentes en el entorno escolar (García-Bacete, Coll, Casares, y Perrin, 2014; Ramos-Díaz, Rodríguez-Fernández, Fernández-Zabala, Revuelta, y Zuazagoitia, 2016; Rodríguez-Fernández, Ramos-Díaz, Madariaga, Arribillaga, y Galende, 2016). Precisamente, un factor que contribuye al funcionamiento óptimo en el entorno educativo es la implicación escolar (school engagement). Esta variable ha sido identificada como un elemento clave para el ajuste y el éxito académico (Motti-Stefadini y Masten, 2013; Ros, Goikoetxea, Gairín, y Lekue, 2012). Un consenso mayoritario en la comunidad científica reconoce la naturaleza multifacética del concepto (Lewis, Huebner, Malone, y Valois, 2011; Wang, Willet, y Eccles 2011), proponiéndose tres dimensiones: cognitiva, emocional y conductual (Appleton, 2012; Christenson, Reschly, y Wylie, 2012; Fredricks, Blumenfeld, y Paris, 2004; González y Verónica-Paoloni, 2015). Cuando los estudiantes se encuentran altamente implicados conductualmente están atentos y participan activamente en cada paso y tarea del proceso de aprendizaje. Cuando están implicados emocionalmente de una manera positiva en una tarea escolar, muestran interés, curiosidad, entusiasmo, confianza y satisfacción con el producto de su aprendizaje. Por último, cuando los estudiantes están altamente implicados cognitivamente son intelectualmente desafiantes y emplean un pensamiento crítico y creativo. En definitiva, la implicación escolar se refleja en los sentimientos, pensamientos y conductas expresadas por el alumnado sobre sus experiencias en el centro educativo (Veiga, Burden, Appleton, Taveira, y Galvao, 2014).

En cualquier caso, aún son escasos los estudios que analizan la posible conexión entre la implicación escolar y la satisfacción con la vida, habiéndose reconocido la existencia de un vacío empírico importante sobre el posible vínculo entre el indicador de bienestar personal y variables educativas cercanas a la implicación escolar tales como el sentimiento de pertenencia o el nivel de vinculación del estudiante con la escuela (Oberle, Schonert-Reichl, y Zumbo, 2011). No obstante, se ha encontrado que bajos niveles de satisfacción con la vida están vinculados con una actitud negativa hacia la escuela y el profesorado (Gilman y Huebener, 2003). Asimismo, en un trabajo longitudinal, los 
investigadores Lewis et al. (2010) encontraron una relación significativa y bidireccional entre satisfacción con la vida e implicación escolar. Por último, un estudio reciente ha encontrado que cuanto más implicados están los estudiantes a nivel escolar, mayor es su percepción de bienestar (Noble y McGrath, 2015).

Además de la información que se ha obtenido sobre cada una de las variables del estudio y sobre sus interrelaciones, también existen indicios del efecto indirecto del apoyo del profesorado sobre la variable satisfacción con la vida, pero teniendo otra variable de ajuste escolar (satisfacción escolar) como variable mediadora de tal efecto (Telef, Arslan, Mert, y Kalafat, 2015). Sin embargo, el análisis conjunto de la satisfacción con la vida a partir de factores contextuales e indicadores de ajuste escolar es limitado. Para una adecuada explicación del funcionamiento óptimo de los estudiantes adolescentes se deben analizar tanto variables ambientales como personales, siendo éste por el camino por el que se precisa avanzar dada la naturaleza multicausal del comportamiento humano (Rodríguez, Droguett, y Revuelta, 2012; Rodríguez et al., 2016). Comprender mejor tanto la dirección como el grado de influencia del apoyo del profesorado y la implicación escolar sobre la satisfacción con la vida permitiría diseñar intervenciones psicoeducativas orientadas a lograr el desarrollo emocional y social del alumnado.

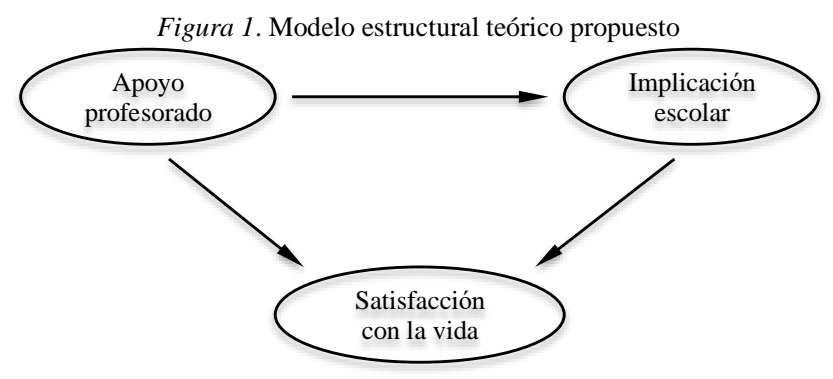

Teniendo en cuenta todo esto, el presente estudio tiene como objetivo someter a prueba un modelo estructural (Figura 1) que hipotetiza: a) la influencia directa del apoyo del profesorado sobre la implicación escolar; b) la influencia directa del apoyo del profesorado sobre la satisfacción con la vida; c) el efecto indirecto positivo de la implicación escolar como elemento mediador entre el apoyo del profesorado y la satisfacción con la vida.

\section{MÉTODO}

\section{Participantes}

Los participantes son elegidos entre los estudiantes de secundaria escolarizados en centros educativos de la Comunidad Autónoma del País Vasco (CAPV). En esta 
investigación se cuenta con un total de 1250 escolares adolescentes de edades comprendidas entre los 12 y los 15 años de edad, de los cuales 612 son chicos (49\%) y 638 chicas $(51 \%)$. Todos ellos son estudiantes de nueve centros escolares ( 5 de titularidad concertada y 4 de titularidad pública) de un nivel sociocultural medio, con una edad media de $13.72(D T=1.09)$. La distribución de los niveles educativos es: $1^{\circ} \mathrm{ESO}, 232(18.6 \%)$; $2^{\circ}$ ESO, 271 (21.8\%); $3^{\circ}$ ESO, 353 (28.2\%) y $4^{\circ}$ ESO, 393 (32.4\%). La ji-cuadrado de Pearson indica que no hay diferencias en la distribución del número de participantes de cada sexo en los diferentes niveles educativos $\left(\chi^{2}(1)=4.66, p>.05\right)$.

\section{Variables e instrumentos de medida}

El apoyo percibido del profesorado es evaluado con el Teacher and Classmate Support Scale del Health Behaviour in School-aged Children (HBSC) (Torsheim, Wold, y Samdal, 2000). El cuestionario está compuesto por 8 ítems agrupados en dos dimensiones: apoyo de los compañeros de clase y apoyo del profesorado. El formato de respuesta es de escala tipo Likert con cinco opciones de respuesta (donde 1=totalmente en desacuerdo y 5=totalmente de acuerdo). En este estudio solo se utiliza la dimensión de apoyo del profesorado, con una consistencia interna de $\alpha=.75$, fiabilidad compuesta $(\mathrm{CFC})=.80$ y varianza media extractada $(\mathrm{AVE})=.51$, que supera el valor de .50 estimado como límite.

Para evaluar la satisfacción con la vida se utiliza la versión castellana, validada por Atienza, Pons, Balaguer y García-Merita (2000) de la Satisfaction with Life Scale (SWLS) de Diener, Emmons, Larsen y Griffin (1985). Mide el juicio global que cada cual hace de su propia vida, con un formato de respuesta en escala Likert de 7 grados desde 1=completamente en desacuerdo a 7=completamente de acuerdo. En la muestra del presente trabajo se extrajo un coeficiente de consistencia interna de $\alpha=.83, \mathrm{CFC}=.83$ y $\mathrm{AVE}=.49$ (el CFC se interpreta del mismo modo que la fiabilidad alfa).

La implicación escolar se calcula a partir de las respuestas al School Engagement Measure (SEM) de Fredericks, Blumenfeld, Friedel, y Paris (2005). El cuestionario está compuesto por 19 ítems a los que se responde en una escala tipo Likert de 5 grados (donde 1=nunca y 5=siempre). A partir de estos 19 ítems los autores obtienen tres factores que miden la implicación conductual, la implicación emocional y la implicación cognitiva, que son las tres medidas que han tomado para este estudio. La evaluación de la fiabilidad ofrece adecuados índices de consistencia interna de los tres factores (Fredricks y McColskey, 2012). Con la muestra del presente estudio, en implicación conductual la consistencia interna de la escala es de $\alpha=.74, \mathrm{CFC}=.83$ y $\mathrm{AVE}=.55$; en implicación emocional la consistencia interna de la escala fue de $\alpha=.81$, $\mathrm{CFC}=.86$ y $\mathrm{AVE}=.55$; y en implicación cognitiva la consistencia interna de la escala fue de $\alpha=.77, \mathrm{CFC}=.89$ y $\mathrm{AVE}=.53$. 


\section{Procedimiento}

Se eligen varios centros al azar a partir de la lista de centros educativos de la Comunidad Autónoma del País Vasco (CAPV) y en cada centro intencionadamente los niveles educativos de interés para este estudio. Después de establecer contacto y realizar los trámites correspondientes con cada institución escolar, se administra la batería de cuestionarios a los participantes dentro del horario lectivo y de manera grupal. Durante el proceso se asegura la participación voluntaria, así como el anonimato de respuestas con el objetivo de reducir la deseabilidad social. Se emplea el criterio de ciego único evitando que los estudiantes conocieran la finalidad del estudio. Ningún estudiante declina su participación en la investigación. Este estudio cumple los valores éticos requeridos en la investigación y la evaluación psicológica respetando los principios fundamentales incluidos en el código ético de la Asociación Americana de Psicología (APA), y en las normativas vigentes (consentimiento informado y derecho a la información, protección de datos personales y garantías de confidencialidad, no discriminación, gratuidad y posibilidad de abandonar el estudio).

\section{Análisis de datos}

El tratamiento de los valores perdidos $(2.1 \%)$ se extrae a partir del algoritmo de maximización de expectativa y de la Cadena Markov Monte Carlo (MCMC) ofrecida por el programa LISREL 8.8. Se eliminan los valores extremos (1.3\%) a través del programa SAS. Para asegurar la normalidad se implementa el método bootstrap correspondiente al programa AMOS 21 (con 2000 repeticiones y estableciendo un intervalo de confianza del $95 \%$ ), que acepta que los resultados de las estimaciones son robustos y, por tanto, no se ven afectados por la falta de normalidad (Byrne, 2001).

Se realiza un análisis correlacional de Pearson entre las variables estudiadas con el objetivo de conocer las posibles conexiones existentes entre ellas y comprobar la no existencia de multicolinealidad. También se lleva a cabo un análisis descriptivo de las medias y desviaciones estándar de todas las variables del estudio. Tanto los estadísticos descriptivos como los coeficientes de correlación son calculados a través del programa SPSS 22. Para la puesta a prueba del modelo de regresión estructural se recurre a la técnica de Modelos de Ecuaciones Estructurales (SEM) proporcionada el programa AMOS 21. Los análisis se efectúan utilizando el procedimiento de máxima verosimilitud, maximun likelihood (ML).

Byrne (2001) propone diversos índices para probar el ajuste del modelo: estadístico ji-cuadrado $\left(\chi^{2}\right)$ y su nivel de probabilidad asociado. Debido a la sensibilidad del estadístico $\chi^{2}$ al tamaño de la muestra y a las desviaciones de la normalidad de los datos (Barret, 2007), se proponen otros índices de ajuste como el GFI (Goodness of Fit Index), CFI (Comparative Fit Index) o TLI (Tucker-Lewis Index), siendo recomendable que todos ellos alcancen valores superiores a .90 (Kline, 2011). Del mismo modo, se 
utilizan los índices RMSEA (Root Mean Square Error of Approximation) y SRMR (Standarized Root Mean Square Residual), cuyos valores inferiores a .08 son indicativos de un ajuste del modelo aceptable, mientras que valores de .05 o menos indican un buen ajuste del modelo (Hu y Bentler, 1999).

\section{RESULTADOS}

\section{Análisis previos}

Tal y como se puede observar en la tabla 1, todas las variables observadas presentan correlaciones significativas entre sí a nivel de $p<.05$ y ninguna supera el nivel de correlación de .90 , indicativo de posible colinealidad entre datos. De hecho, la correlación más alta alcanza el valor $r=.585$. El cuarto ítem de apoyo del profesorado (AP4) alcanza los índices más altos de correlación con la escala de implicación emocional $(r=.398, p>.05)$ y el segundo ítem de satisfacción con la vida (SV2) (r=.248, $p>.05)$. Por último, es la escala de implicación emocional la que registra nuevamente mayores índices de correlación con dos ítems de satisfacción con la vida: SV2 $(r=.372, p>.05)$ y SV5 $(r=.485, p>.05)$.

Tabla 1. Matriz de Correlaciones entre las Variables Observadas, Medias y Desviaciones Típicas

\begin{tabular}{|c|c|c|c|c|c|c|c|c|c|c|c|c|}
\hline Variables & AP1 & AP2 & AP3 & $\mathrm{AP} 4$ & ICDT & IEMO & ICOG & SV1 & SV2 & SV3 & SV4 & SV5 \\
\hline AP1 & 1 & $.440 * *$ & $349 * *$ & $.401 * *$ & $.183^{* *}$ & $.307 * *$ & $191 * *$ & $145 * *$ & $196^{* *}$ & $157 * *$ & $142 * *$ & $134 * *$ \\
\hline $\mathrm{AP} 2$ & & 1 & $.405^{* *}$ & $.418^{* *}$ & $.328 * *$ & $.379^{* *}$ & $.244 * *$ & $200 * *$ & $146^{* *}$ & $.173^{* * *}$ & $.213^{* *}$ & $.148^{* *}$ \\
\hline AP3 & & & 1 & $.555^{*}$ & $.324 * *$ & $.337 * *$ & $.205 * *$ & $.167 * *$ & $.151 * *$ & $.192 * *$ & $.187 * *$ & $.125 * *$ \\
\hline AP4 & & & & 1 & $.341 * *$ & $.398^{* *}$ & $.284 * *$ & $.172 * *$ & $.187 * *$ & $.221 * *$ & $.248 * *$ & $.141^{* *}$ \\
\hline ICDT & & & & & 1 & $.485^{* *}$ & $.390 * *$ & $.249 * *$ & $214 * *$ & $.265^{* *}$ & $249 * *$ & $181 * *$ \\
\hline IEMO & & & & & & 1 & $.429 * *$ & $.273 * *$ & $.372 * *$ & $.327 * *$ & $.284 * *$ & $.485^{* *}$ \\
\hline ICOG & & & & & & & 1 & $.205 * *$ & $.169 * *$ & $.154 * *$ & $.212 * *$ & $.131 * *$ \\
\hline SV1 & & & & & & & & 1 & $.529 * *$ & $.561 * *$ & $.509 * *$ & $.447 * *$ \\
\hline SV2 & & & & & & & & & 1 & $.585^{* *}$ & $.394 * *$ & $.457 * *$ \\
\hline SV3 & & & & & & & & & & 1 & $.573 * *$ & $.569 * *$ \\
\hline SV4 & & & & & & & & & & & 1 & $.475^{* *}$ \\
\hline SV5 & & & & & & & & & & & & 1 \\
\hline $\mathrm{M}$ & 3.47 & 3.31 & 3.44 & 3.37 & 3.86 & 3.63 & 2.85 & 4.93 & 5.54 & 5.73 & 5.34 & 4.82 \\
\hline D.T & 1.04 & 1.04 & 1.10 & 1.07 & .58 & .67 & .71 & 1.29 & 1.26 & 1.29 & 1.36 & 1.91 \\
\hline
\end{tabular}

\section{Análisis del modelo teórico}

Antes de estimar el modelo estructural, se lleva a cabo un análisis factorial confirmatorio (AFC) de los modelos de medida para cada una de las escalas utilizadas (HBSC, SWL y SEM).

Respecto al modelo de medida del apoyo del profesorado, se obtienen índices satisfactorios de ajuste a los datos $\left(\chi^{2}{ }_{(1)}=2.218, p=.136\right.$; GFI=.999; CFI=.999; TLI=.994; 
SRMR=.006; RMSEA=.031) tras la incorporación de una covarianza de error con un cambio en el parámetro estandarizado de .22 .

En relación al modelo de medida de la satisfacción con la vida, los resultados del AFC realizado indican un buen ajuste a los datos empíricos $\left(\chi^{2}(4)=13.54, p<.001\right.$; $\mathrm{GFI}=.996 ; \mathrm{CFI}=.996 ; \mathrm{TLI}=.990 ; \mathrm{SRMR}=.013$; $\mathrm{RMSEA}=.044)$ tras la incorporación de una covarianza de error con un cambio en el parámetro estandarizado de .24 .

Por último, el AFC al que se somete la estructura tridimensional de la implicación escolar proporciona índices de ajuste adecuados $\left(\chi_{(100)}^{2}=676.93, p<.001\right.$; $\mathrm{GFI}=.931 ; \mathrm{CFI}=.906 ; \mathrm{SRMR}=.058$; RMSEA=.068) tras la eliminación de tres ítems por baja carga factorial y el establecimiento de una covarianza de error con un cambio en el parámetro estandarizado de .16. Aunque el valor de ji-cuadrado de los dos últimos modelos de medida presentan asociada una $p<.001$ y por tanto induce a rechazar la hipótesis nula de que el modelo ajusta a los datos empíricos, el hecho de que el resto de parámetros hayan ofrecido valores adecuados, permite afirmar que ambos modelos de medida ajustan correctamente a los datos.

Una vez analizados los modelos de medida, se estima el modelo teórico mostrado en la figura 1. En él se incluyen 3 variables latentes cuyos indicadores, en el caso de las variables unidimensionales de apoyo del profesorado y satisfacción con la vida son los ítems de las escalas del test correspondiente y, en el caso de la variable tridimensional implicación escolar (implicación conductual, implicación emocional e implicación cognitiva), los indicadores son las puntuaciones obtenidas por los sujetos en las distintas escalas (parcels).

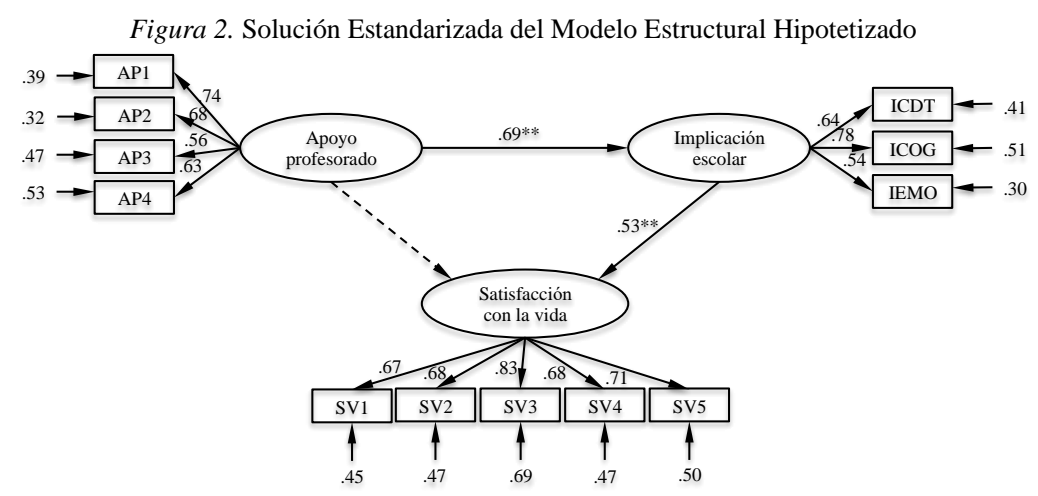

Se somete a prueba el modelo hipotético inicial (Figura 1), que propone que el apoyo del profesorado estaría positivamente relacionado con la implicación escolar, la cual, a su vez, tendría capacidad predictiva sobre la satisfacción con la vida, ejerciendo por tanto, un rol mediador entre el apoyo del profesorado y dicho indicador de bienestar 
personal. Además, se plantea una vía directa desde el apoyo del profesorado hacia la satisfacción con la vida. Un primer análisis de los parámetros resultantes indica que el modelo planteado inicialmente (Figura 2) ajusta correctamente a los datos empíricos: $\left(\chi^{2}(51)=219.743, p<.001 ; \mathrm{GFI}=.972 ; \mathrm{CFI}=.965 ; \mathrm{TLI}=.954 ; \mathrm{SRMR}=.031 ; \mathrm{RMSEA}=.051\right)$.

Tal y como puede observarse, el apoyo del profesorado se asocia significativamente con la implicación escolar y ésta con la satisfacción con la vida. Para demostrar la significación de la mediación, de emplea el test de Sobel (1982). Los resultados de este test indican que la implicación escolar media significativamente entre el apoyo del profesorado y la satisfacción con la vida $(z=420.31, p<.01)$.

\section{Efectos directos e indirectos entre las variables estudiadas}

$\mathrm{Si}$ se analizan individualmente los coeficientes de regresión del modelo hipotetizado (Tabla 2), se observa que todas las vías directas propuestas obtienen significación a un nivel $p<.01$ excepto el par apoyo del profesorado-satisfacción con la vida $(\beta=.004, p>.01)$. De este modo, el apoyo del profesorado muestra una varianza observada del $69 \%$ de la implicación escolar $(\beta=.688, p<.01)$. En cuanto a la satisfacción con la vida, se observa que dicha variable viene explicada de forma directa por la implicación escolar $(\beta=.528, p<.01)$.

En lo que respecta a los efectos indirectos sobre la variable satisfacción con la vida, cuya proporción de varianza explicada es el 53\%, los resultados indican que el apoyo del profesorado presenta un efecto significativo de forma indirecta, mediado por el nivel más o menos positivo de la implicación escolar $(\beta=.413, p<.01)$.

Tabla 2. Efectos Directos e Indirectos Estandarizados entre las Variables Estudiadas

\begin{tabular}{|c|c|c|c|c|c|}
\hline Coeficiente & & & & $\begin{array}{l}\text { Efecto } \\
\text { directo }\end{array}$ & $\begin{array}{c}\text { Efecto } \\
\text { indirecto }\end{array}$ \\
\hline Apoyo profesorado & $\longrightarrow$ & Implicación escolar & & $.688 * *$ & --- \\
\hline Implicación escolar & $\longrightarrow$ & Satisfacción con la vida & & $.528 * *$ & --- \\
\hline Apoyo profesorado & $\longrightarrow$ & Implicación escolar $\longrightarrow$ & Satisfacción con la vida & --- & $.363 * *$ \\
\hline
\end{tabular}

En síntesis, las comprobaciones más relevantes obtenidas son las que se especifican a continuación: por un lado, el apoyo del profesorado está relacionado con la satisfacción con la vida de manera exclusivamente indirecta a través de la implicación escolar, ya que el efecto directo del apoyo del profesorado sobre la satisfacción con la vida no resulta significativo. En consecuencia, puede afirmarse que el apoyo del profesorado activa la implicación escolar $\left(R^{2}=.69\right)$ como variable mediadora, que a su vez incide directamente junto con la influencia del apoyo del profesorado sobre la satisfacción con la vida del alumnado $\left(R^{2}=.53\right)$. 


\section{DISCUSIÓN Y CONCLUSIONES}

La investigación psicoeducativa actual apuesta por analizar el comportamiento adaptativo de los estudiantes adolescentes en el contexto educativo desde un enfoque multicausal que agrupe variables, tanto de carácter psicológico, como ambiental (ver Moreno y Vera, 2011; Rodríguez-Fernández et al., 2012; Rodríguez-Fernández et al., 2016; Rodríguez-Fernández et al., en prensa; Rodríguez-Fernández, Ramos-Díaz, Ros, y Zuazagoitia, en prensa; Telef et al., 2015).

En esta misma línea, el presente trabajo ha proporcionado evidencia empírica acerca de la influencia directa que ejerce el apoyo del profesorado percibido por parte del alumnado sobre la varianza observada de implicación escolar. Igualmente, se ha constatado que, a través de la implicación escolar, el apoyo del profesorado afecta indirectamente a la satisfacción con la vida de los escolares adolescentes. Los resultados obtenidos en este estudio confirman que la relación más significativa en el modelo planteado es la que mantiene el apoyo del profesorado con la implicación escolar, apareciendo dicha implicación como una variable de adaptación escolar decisiva en la predicción de la satisfacción con la vida durante la adolescencia. Asimismo, se demuestra la función mediadora que ejerce la implicación escolar entre el contexto escolar del adolescente y su ajuste psicológico.

El ajuste psicológico del alumnado adolescente depende, en gran medida, de la interacción de las características psicológicas con aspectos ambientales de su entorno (Proctor et al., 2009). Dentro del ambiente en el que se encuentran inmersos los estudiantes, se ha demostrado la relevancia de los sistemas más próximos en su implicación escolar (Estell y Perdue, 2013), destacando el apoyo del profesorado frente a otras fuentes de soporte social como la familia y los iguales (Rodríguez-Fernández et al., en prensa). A pesar de que la relevancia del apoyo del profesorado en la predicción del bienestar personal y escolar ha sido corroborada (Danielsen, Wiium, Wilhelmsen, y Wold, 2010; Guess y McCane-Bowling, 2016), quedaba por analizar la posible incidencia del indicador de adaptación escolar sobre la percepción de satisfacción con la vida en la adolescencia. Del mismo modo, apenas se encuentran referencias de modelos que consideren la adaptación escolar como una variable mediadora entre el contexto y la felicidad de los escolares adolescentes, a excepción del estudio de Telef et al. (2015). Los resultados del presente trabajo vienen a confirmar en parte la investigación previa, ya que (a) aportan información sobre la influencia del apoyo del profesorado sobre la implicación escolar, pero niegan que el apoyo del profesorado tenga un efecto directo sobre la satisfacción con la vida al seguir una ruta indirecta: contribuye a la implicación escolar y ésta a su vez en la satisfacción con la vida; y (b) confirman la influencia de la implicación escolar como variable mediadora entre el apoyo del profesorado y la satisfacción con la vida. 
La corroboración del peso de la influencia del apoyo del profesorado tanto en el éxito escolar como en el bienestar del estudiante puede tratarse de un dato relevante, debido a que ofrece nueva información acerca de la importancia que tiene el establecimiento de vínculos de apoyo saludables con la figura docente para que los adolescentes se impliquen activamente con el centro educativo, lugar en el que invierten una gran cantidad de horas. Precisamente, el hecho de sentirse a gusto en la escuela durante esas horas tiene una gran relevancia sobre la percepción de satisfacción con la vida. A su vez, el ajuste escolar promovido por el apoyo del profesorado influirá en el aumento de cogniciones satisfactorias sobre la trayectoria vital de los adolescentes.

No obstante, a pesar de lo novedoso de los datos, este trabajo también presenta ciertas limitaciones y deja abiertas propuestas interesantes para futuros estudios. En primer lugar, los resultados presentados se refieren únicamente a estudiantes de secundaria de 12 a 15 años de edad. En consecuencia, el grupo de la muestra utilizada en este estudio podría ampliarse para la generalización de los resultados en poblaciones que se sitúan fuera de este intervalo de edad y nivel educativo. Sería interesante que futuros estudios implementaran un análisis multimuestra en función del nivel educativo (ESO, Primaria, Bachiller, Universidad, etc.) a fin de conocer si este modelo explica dichas relaciones de manera estable a lo largo de la trayectoria educativa, lo que permitiría poner a prueba de forma más estricta el modelo obtenido. Además, ante la utilización de medidas exclusivamente autoinformadas se presenta la necesidad de incluir en futuras investigaciones variables medidas de forma objetiva que completen el análisis del modelo planteado, como por ejemplo el rendimiento académico. Ésta es la mayor limitación, ya que es de esperar que el apoyo percibido del profesorado mejore la implicación del estudiante, y esta mayor implicación lleve a un mejor rendimiento académico que pueda contribuir incluso a una mayor satisfacción con la vida. Otra limitación es el carácter transversal del estudio, que hace que no se puedan probar las relaciones de causalidad planteadas en el modelo. Por otro lado, sería asimismo interesante que futuros estudios puedan incluir otras fuentes de apoyo social (familia, círculo de amistades, compañeros de clase, etc.) para realizar un análisis diferencial de la influencia que las distintas figuras de soporte en la adolescencia sobre la implicación escolar y la satisfacción con la vida.

A pesar de estas limitaciones, los resultados del presente trabajo poseen implicaciones educativas a tener en cuenta en el diseño de intervenciones psicoeducativas encaminadas a la potenciación de la implicación escolar y al ajuste psicológico del estudiante. Conocer la naturaleza multicausal de ambas variables (implicación y satisfacción con la vida) permitirá el desarrollo de programas psicoeducativos encaminados a potenciar las relaciones profesorado-alumnado principalmente debido al papel de protección y apoyo social que ofrecería el profesorado en determinados momentos y ante determinados eventos académicos complicados para el alumnado. Entre los comportamientos de los profesores que favorecen su apoyo a la autonomía de los 
estudiantes destacan ser menos controlador, querer apoyar la autonomía y aprender habilidades interpersonales para fomentar un estilo de apoyo a la autonomía (Reeve, 2009). Investigaciones en la línea del presente trabajo redundan en el diseño de programas de desarrollo profesional del profesorado orientados al ajuste escolar (Guay, Valois, Falardeau, y Lessard, 2016). Ahora bien, también es importante hacer esfuerzos por mejorar la implicación escolar más allá de esta forma indirecta (a través del apoyo del profesorado) y realizar un trabajo directo sobre la misma, tratando de modificar los sentimientos, conductas y pensamientos que el alumno mantiene con respecto a su proceso de aprendizaje.

\section{Agradecimientos}

Las autoras del presente estudio forman parte del Grupo Consolidado de Investigación IT701-13 del Sistema Universitario Vasco. El estudio se llevó a cabo como parte del proyecto de investigación EHUA 14/10 en la Universidad del País Vasco (UPV-EHU).

\section{REFERENCIAS}

Appleton, J.J. (2012). Systems consultation: Developing the assessment-to-intervention link with the Student Engagement Instrument. En S.L. Christenson, A.L. Reschly, C. Wylie, S.L. Christenson, A.L. Reschly y C. Wylie (Eds.), Handbook of research on student engagement (pp. 725-741). New York, NY, US: Springer. doi:10.1007/978-1-4614-20187_35

Atienza, F., Pons, D., Balaguer, I., y García-Merita, M. (2000). Propiedades psicométricas de la escala de satisfacción con la vida en adolescentes. Psicothema, 12(2), 314-319.

Azpiazu, L., Esnaola, I., y Sarasa, M. (2015). Capacidad predictiva del apoyo social en la inteligencia emocional de adolescentes. European Journal of Education and Psychology, 8(1), 23-29. doi:10.1016/j.ejeps.2015.10.003

Barrett, P. (2007). Structural equation modelling: Adjudging model fit. Personality and Individual Differences, 42(5), 815-824. doi:10.1016/j.paid.2006.09.018

Byrne, B.M. (2001). Structural equation modeling with AMOS, EQS, and LISREL: Comparative approaches to testing for the factorial validity of a measuring instrument. International Journal of Testing, 1(1), 55-86. doi:10.1207/S15327574IJT0101_4

Christenson, S.L., Reschly, A.L., y Wylie, C. (2012). Handbook of research on student engagement. New York, NY, US: Springer Science. doi:10.1007/978-1-4614-2018-7

Campbell, A., Converse, P.E., y Rodgers, W.L. (1976). The quality of American life: Perceptions, evaluations, and satisfactions. New York, NY US: Russell Sage Foundation.

Casas, F. (2011). Subjective social indicators and child and adolescent well-being. Child Indicators Research, 4(4), 555-575. doi:10.1007/s12187-010-9093-z

Chu, P.S., Saucier, D.A., y Hafner, E. (2010). Meta-analysis of the relationships between social support and well-being in children and adolescents. Journal of Social and Clinical Psychology, 29(6), 624.

Cohen, S. (2004). Social relationships and health. American Psychologist, 59(8), 676-684. doi:10.1037/0003-066X.59.8.676 
Diener, E. (1994). Assessing subjective well-being: Progress and opportunities. Social Indicators Research, 31(2), 103-157.

Diener, E., Emmons, R., Larsen, R.J., y Griffin, S. (1985). The Satisfaction With Life Scale. Journal of Personality Assessment, 49, 71-75.

Diener, E., Oishi, S., y Lucas, R.E. (2009). Subjective well-being: The science of happiness and life satisfaction. En S.J. Lopez, C.R. Snyder, S.J. Lopez, C.R. Snyder (Eds.), Oxford handbook of positive psychology (pp. 187-194). New York, NY, US: Oxford University Press.

Danielsen, A.G., Wiium, N., Wilhelmsen, B.U., y Wold, B. (2010). Perceived support provided by teachers and classmates and students' self-reported academic initiative. Journal of School Psychology, 48(3), 247-267. doi:10.1016/j.jsp.2010.02.002

Estell, D.B., y Perdue, N.H. (2013). Social support and behavioral and affective school engagement: The effects of peers, parents, and teachers. Psychology in the Schools, 50(4), 325-339. doi:10.1002/pits.21681

Fernández-Zabala, A., Goñi, E., Camino, I., y Zulaika, L. (2016). Family and school context in school engagement. European Journal of Education and Psychology, 9(2). doi:10.1016/j.ejeps.2015.09.001

Fredricks, J.A., Blumenfeld, P.C., Friedel, J., y Paris, A. (2005). School engagement. En K. A. Moore y L. Lippman (Eds.), Conceptualizing and measuring indicators of positive development: What do children need to flourish (pp. 305-321). New York: Kluwer Academic/Plenum Press.

Fredricks, J.A., Blumenfeld, P.C., y Paris, A.H. (2004). School engagement: Potential of the concept, state of the evidence. Review of Educational Research, 74(1), 59-109. doi:10.3102/00346543074001059

Froh, J.J., Huebner, E.S., Youssef, A.J., y Conte, V. (2011), Acknowledging and appreciating the full spectrum of the human condition: School Psychology's (limited) focus on positive psychological functioning. Psychology in the Schools, 48(2), 110-123. doi: $10.1002 /$ pits. 20530

Fuentes, M.C., García, F., Gracia, E., y Alarcón, A. (2014). Los estilos parentales de socialización y el ajuste psicológico. Un estudio con adolescentes españoles. Revista de Psicodidáctica, 20(1), 117-138. doi: 10.1387/RevPsicodidact.10876

García-Bacete, F.J.G., Coll, P.F., Casares, M.I.M., y Perrin, G.M. (2014). Las relaciones del profesorado con el alumnado en aulas del ciclo inicial de Educación Primaria. Adaptación del Questionnaire on Teacher Interaction-Early Primary (QTI-EP). Revista de Psicodidáctica, 19(1), 211-231. doi:10.1387/RevPsicodidact.9081

García-Moya, I., Brooks, F., Morgan, A., y Moreno, C. (2014). Subjective well-being in adolescence and teacher connectedness: A health asset analysis. Health Education Journal, 74(6), 641654. doi: 10.1177/0017896914555039

Gilman, R., y Huebner, E.S. (2003). A review of life satisfaction research with children and adolescents. School Psychology Quarterly, 18, 192-205.

González, A., y Paolini, P. (2015). Implicación y rendimiento en Física: el papel de las estrategias docentes en el aula, y el interés personal y situacional. Revista de Psicodidáctica, 20(1). doi:http:10.1387/RevPsicodidact.11370

Guay, F., Valois, P., Falardeau, E., y Lessard, V. (2016). Examining the effects of a professional development program on teachers' pedagogical practices and students' motivational resources and achievement in written French. Learning and Individual Differences, 45, 291-298. doi:10.1016/j.lindif.2015.11.014

Guess, P.E., y McCane-Bowling, S.J. (2016). Teacher support and Life satisfaction: An investigation with urban, middle school students. Education and Urban Society, 48(1), 30-47. doi: $10.1177 / 0013124513514604$ 
Hu, L., y Bentler, P.M. (1999). Cutoff criteria for fit indexes in covariance structure analysis: Conventional criteria versus new alternatives. Structural Equation Modeling, 6(1), 1-55. doi:10.1080/10705519909540118

Kline, R.B. (2011). Principles and practice of structural equation modeling. New York, NY, US: Guilford Press.

Kristjánsson, K. (2012). Positive psychology and positive education: Old wine in new bottles? Educational Psychologist, 47(2), 86-105. doi: 10.10080/00461520.2011.610678

Lakey, B., y Scoboria, A. (2005). The relative contribution of trait and social influences to the links among perceived social support, affect, and self-esteem. Journal of Personality, 73(2), 361-388. doi: 10.1111/j.1467-6494.2005.00312.x

Lewis, A.D., Huebner, E., Malone, P.S., y Valois, R.F. (2011). Life satisfaction and student engagement in adolescents. Journal of Youth and Adolescence, 40(3), 249-262. doi:10.1007/s10964-010-9517-6

Moreno, J.A., y Vera, J.A. (2011). Modelo causal de la satisfacción con la vida en estudiantes adolescentes. Revista de Psicodidáctica, 16(2), 367-380.

Motti-Stefanidi, F., y Masten, A.S. (2013). School success and school engagement of immigrant children and adolescents: A risk and resilience developmental perspective. European Psychologist, 18(2), 126-135. doi:10.1027/1016-9040/a000139

Noble, T., y McGrath, H. (2015). PROSPER: A new framework for positive education. Psychology of Well-Being, 5(1), 1-17. doi:10.1186/s13612-015-0030-2

Oberle, E., Schonert-Reichl, K.A., y Zumbo, B.D. (2011). Life satisfaction in early adolescence: Personal, neighborhood, school, family, and peer influences. Journal of Youth and Adolescence, 40(7), 889-901.

Pertegal, M.Á., Oliva, A., y Hernando, A. (2010). Los programas escolares como promotores del desarrollo positivo adolescente. Cultura y Educación, 22(1), 53-66. doi:10.1174/113564010790935169

Proctor, C.L., Linley, P.A., y Maltby, J. (2009). Youth life satisfaction: A review of the literature. Journal of Happiness Studies, 10(5), 583-630. doi:10.1007/s10902-008-9110-9

Ramos-Díaz, E., Rodríguez-Fernández, A., Fernández-Zabala, A., Revuelta, L., y Zuazagoitia, A. (2016). Apoyo social percibido, autoconcepto e implicación escolar de estudiantes adolescentes. Revista de Psicodidáctica, 21(2). doi: 10.1387/RevPsicodidact.14848

Reeve, J. (2009). Why teachers adopt a controlling motivating style toward students and how they can become more autonomy supportive. Educational Psychologist, 44(3), 159-175. doi: 10.1080/00461520903028990.

Rodríguez-Fernández, A., y Goñi-Grandmontagne, A. (2011). La estructura tridimensional del bienestar subjetivo. Anales de Psicología, 27(2), 327-332.

Rodríguez-Fernández, A., Ramos-Díaz, E., Madariaga, J.M., Arribillaga, A., y Galende, N. (2016). Steps in the construction and verification of an explanatory model of psychosocial adjustment. European Journal of Education and Psychology, 9(1). http://dx.doi.org/10.1016/j.ejeps.2015.11.002

Rodríguez-Fernández, A., Ramos-Díaz, E., Ros, I., y Zuazagoitia, A. (en prensa). Implicación escolar de estudiantes de secundaria: La influencia de la resiliencia, el autoconcepto y el apoyo social percibido. Educación XX1 (2016). doi:10.5944/educxx1.16026

Ros, I., Goikoetxea, J., Gairín, J., y Lekue, P. (2012). Implicación del alumnado en la escuela: diferencias interindividuales e intercentros. Revista de Psicodidáctica, 17(2), 291-307. doi: 10.1387/Rev.Psicodidact.4496

Sobel, M.E. (1982). Asymptotic intervals for indirect effects in structural equations models. En S. Leinhart (Ed.), Sociological methodology (pp. 290-312). San Francisco: Jossey-Bass. 
Telef, B.B., Arslan, G., Mert, A., y Kalafat, S. (2015). The mediation effect of school satisfaction in the relationship between teacher support, positive affect and life satisfaction in adolescents. Educational Research and Reviews, 10(12), 1633-1640. doi: 10.5897/ERR2015.2282

Tennant, J.E., Demaray, M.K., Malecki, C.K., Terry, M.N., Clary, M., y Elzinga, N. (2014). Students' ratings of teacher support and academic and social-emotional well-being. School Psychology Quarterly, 30(4), 494-512. doi: 10.1037/spq0000106

Torsheim, T., Wold, B., y Samdal, O. (2000). The Teacher and Classmate Support scale: Factor structure, test-retest reliability and validity in samples of 13- and 15-year-old adolescents. School Psychology International, 21(2), 195-212. doi:10.1177/0143034300212006

Veiga, F.H., Burden, R., Appleton, J., Taveira, M.D.C., y Galvao, D. (2014). Envolvimiento de los estudiantes en la escuela: conceptualización y relaciones con variables personales y rendimiento académico. Una revisión de la literatura. Revista de Psicología y Educación, 9(1), 29-47.

Wang, M.T., Willett, J.B., y Eccles, J.S. (2011). The assessment of school engagement: Examining dimensionality and measurement invariance by gender and race/ethnicity. Journal of School Psychology, 49(4), 465-480. doi:10.1016/j.jsp.2011.04.001

Recibido: 30 de abril de 2016 Recepción Modificaciones: 15 de julio de 2016 Aceptado: 18 de julio de 2016 